\title{
Analysis of Various Deep learning Algorithms for Tamil Character Recognition
}

\author{
Dr.L.Ashok Kumar ${ }^{1}$, J.Shalini ${ }^{2}$, Dr.Karthika Renuka $\mathrm{D}^{3}$ \\ \{lak.eee@psgtech.ac.in ${ }^{1}$, Shalinijayabal1997@gmail.com ${ }^{2}$,dkr.it@psgtech.ac.in $\left.{ }^{3}\right\}$ \\ Professor,Department of EEE,PSG College of Technology,Coimbatore, India ${ }^{1}$, Project \\ Associate,Department of EEE,PSG College of Technology,Coimbatore, India ${ }^{2}$ Associate Professor \\ ,Department of IT,PSG College of Technology,Coimbatore, India ${ }^{3}$
}

\begin{abstract}
Process of converting Handwritten Tamil letters into editable form for extracting the information present in documents, Invoices that in handwritten letters that are difficult to find out the word present in the document because of bad handwriting and poor quality of the picture, Social media comments for analyzing and extracting the sentiment behind the text. Nowadays people started to deliver their thoughts through their own language Native Tamil. People find it easy to post thighs in Tamil which also leads to false information, violated messages, and cyberbullying. To address this, the notion of deep learning algorithms is implemented to classify the letters in digital form and also to suggest that the combination of letters obtained from deep learning results in better performance and good accuracy as well. The method involves the identification of optical characters to obtain feedback for the model and the creation of a model using neural networks which include CNN, Yolo and ResNet. Existing works involves using a simple OCR system but it results in a low-level recognition rate it finds difficult blurred images, shaken images, slightly tilted image's, poor quality images to recognize the text and give it back as an editable form to overcome this above situation selecting these models for testing with the Tamil character's to choose upon the best fit model to proceed for the future work of increasing the dataset and to recognize all the combination of words given in the input source of Tamil letters. The dataset used here is from HPLabs India, which provides a large dataset of around 60,000 samples of isolated Tamil letters collected from both male and female candidates with different age groups. Tamil text classification is generally used to classify particular letters from handwritten letters in the digital form of images.
\end{abstract}

Keywords: Deep learning, Convolutional neural network, Yolo, ResNet, Machine Learning, Character Recognition.

\section{Introduction}

In present days obtaining information and altering the content in pictures that are present within background images are time consuming. The Optical Character Recognition (OCR) idea emerges to take care of this issue. OCR works primarily utilizing Artificial Intelligence calculation and is significant in developing and exploring man-made brainpower. Optical character acknowledgment permits to change over the characters in printed archives, computerized pictures, and examined records to the word group. The conventional method for entering the information of the printed reports, checked records and pictures into the PC is 
through a console which is inefficient when there is a huge volume of information. OCR is utilized to enter the information from those records electronically without the intercession of people.

OCR technology was originally developed to recognize printed text; it can also be used for handwrittentextrecognition and verification. For instance, OCR software is used by postal services to automatically process letters and packages based on the address. To validate the mailing address, the algorithm checks the scanned information against a database of existing addresses. One of the daunting tasks for optical character recognition is Tamil character recognition (TCR). It is very difficult to identify handwritten Tamil characters, due to variations in scale, style, and angle of orientation. Time-consuming and poor accuracy of character editing and reprinting of text documents printed on paper. It is used to identify and transform the characters from the scanned digital input image into a machine-editable form. Due to its different writing styles and large numbers of characters, the optical handwriting recognition system produced for the Tamil language has a very low recognition rate.

\section{Related work}

A convolutional neural network for proposing in offline handwritten Tamil characters was developed with a large number of datasets [1] it sets a benchmark for character recognition HTCR using the deep learning technique which includes the convolutional neural network for feature extraction, train the model. Using CNN as the model produces an accuracy of $95.16 \%$ in result analysis through the entire model description. It improves character recognition by introducing neural networks into the system [2]. That by using the CNN that recognizes the online Tamil characters. The Fourier descriptor is combined with the back propagation network; it gives a good recognition rate of $95 \%$ accuracy for handwritten Tamil letters. The construction for handwritten character popularity system using convolutional neural network [3] which will be capable of understanding the handwritten characters and provide the outputs using the training dataset they used IAM dataset to train the model. The neural community consists of several layers which assist the education procedure.

Using Tamil characters for recognition the text also includes digits in them a mixed form of words contain digits[4], the system explains architecture for the model will contain the convolutional model and after the process of recognition that takes place, it sends to the Unicode mapping process to convert the letters into computerized forms. For proposing a new algorithm Back propagation algorithm along with Fourier Descriptors was developed to overcome the text classification time consumption [5]. Definition of Back propagation states tuning the weights of an error rate that obtained in the previous epochs (or) iterations. The dataset they collected was formed IWFHR 2006, a competition held in France for handwriting recognition that contains more images that are collected from both male and female participants of different ages.

Hybrid decision tree methods were introduced for Tamil text classification along with the problem states of analyzing the text present in the given input of images [6] it also contains multi-class classification to recognize Tamil characters using binary support vector machines (SVMs) organized in a hybrid decision tree. Techniques used to classify the text in Tamil are OCR, SVM, and the hybrid tree. Supervised the learning technique with the support vector machine [7] and which can be applied to the blur images on the dataset. Dataset of HP labs 
India dataset that contains an average of 60,000 images of isolated Tamil letters with different handwriting was used. The superior performance of SVM is because of the superior generalization capability of the support vector machines. Artificial network technique to recognize the Tamil characters [8], a technique that is carried out is Kohonen SOM technique to go through the process, results in increasing the accuracy rate by increasing the dataset count and gives the output of predicting the word present in the input raw file.

Approach to OCR using the image recognition based classification for the ancient Tamil Inscriptions in Temples [9] used the dataset of Ancient script handwriting dataset to train the model. The output obtained could not be digitally segmented due to the lack of availability of any language parser for ancient Tamil scripts. To develop efficient systems for recognizing the Indian languages, especially for Tamil, a south Indian language that is widely used in Tamilnadu, Srilanka, and Pudhucherry, Singapore [10]. For dataset collection, they included the images of letters that are written and scanned. Neural classifiers feed forward neural networks are used for the classification of Tamil characters. Recognition of Tamil characters using an approach of VGG16 in deep learning[11] using VGG16(Visual Geometry Group) with the combination of convolutional neural network that it was trained on more than a million images on image net database on which it contains 16 layers of feed forward deep and it can be classified into about 1000 objects. In transfer learning, the network will be pretrained with a certain set of datasets concerning the application.

Discriminative auto encoder for feature extraction for the character recognition application [12], stacked architecture with the basic discriminating auto encoder as a unit to extract related representation from the data from the training. With even simple classification systems such as KNN, the efficiency of our feature extraction algorithm ensures high classification accuracy (K-nearest neighbor, text feature extraction based upon the stack variational auto-encoder [13]. To minimize noise interference and improve robustness and feature discrimination of the model, a noise reduction mechanism is built for the variational auto encoder in the input layer of text feature extraction. Three kinds of deep SAVE network architectures are designed to enhance the ability to reflect in-depth learning to mine feature intention.

Digital recognition of handwritten letters using Resnet provides an efficient approach to the model [14], suggested approach incorporates Bayesian inference into the existing ResNet18 framework to reduce uncertainty in handwritten digit recognition when a new class of test digit is introduced. Isolated Bangali handwritten character recognition states the Resnet architecture is more suitable than all the other Deep learning algorithms with an alternative of the VGG-16 model [15]. The study states a modified ResNet-18 architecture (Convolutional neural network architecture) is used to recognize Bangla handwritten letters. The proposed strategy is used for two isolated Bangla handwritten datasets that were newly developed. It attains state-of-the-art recognition performance using the proposed technique.

Character recognition using these algorithms work well but some are not suitable for large datasets and with a large number of classes. The system has to be scalable that holds a term of giving high accuracy, prediction rate, should not take much time to train and test, by reviewing all the above and in the Introduction part, the below concepts and the implementation will cover the best algorithm that is suitable for perdition with a large number of dataset CNN, OCR, Yolo, and ResNet. Using these algorithms by implementing all with the 
same dataset and by changing the model accordingly, the analysis is to be taken to decide which model is the best fit for the future proposal.

\section{Character recognition models}

\section{A. Convolutional Neural Networks}

A convolutional neural network with Imagedatagenerator works under Keras by which it augments the images while the model is training in real-time. Using the CNN model by which it consumes a less amount of memory because it would not uses the class concept here it generates the classes according to the folders that were mentioned as a batch so it consumes less amount memory to the process of predicting the class labels for given input image used to generate a more number of images, shifting, rotation, slips Fig. 1 illustrates the CNN model for character recognition. By using this concept it yields more accuracy under a short period of implementation compared to all deep learning methods. Using a convolutional neural network splits the dataset set into two testing and training in the ratio of 80:20 for testing and training. Inside each folder, it contains a separate file folder in the name of class labels. The dataset was collected and given into the class folders according to the letters taken for pre-processing.

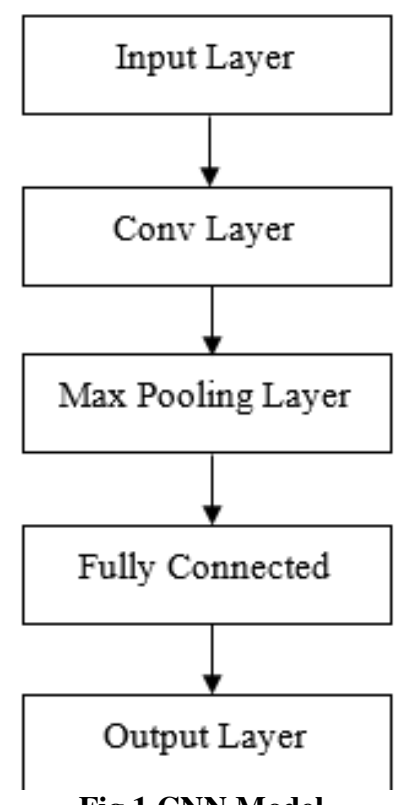

\section{B.Yolo}

Fig.1 CNN Model

Yolo is a pre-trained model that contains very simple steps to follow up the input data that had been divided into $\mathrm{S} \times \mathrm{S}$ grid dimensions where the object that appears in a particular grid that cells are responsible for the object detection. The Bounding box that highlights an object with some parameters that width (bw), height (bh), Class of an Object, Bound box center will be represented as (bx, by). Intersection of the union works as a box for the overlap of the 
output that surrounds the object perfectly to detect the object. The values on IOU will be equal to 1 if the predicted bounded box value is the same as the real box values it eliminates the unnecessary which are not useful for the detection in the IOU part. To train the dataset by consuming less amount of time with good efficiency Yolo holds a good review in Tamil character recognition. Using Yolo to the system, developing a boundary box Fig. 2 explains the Yolo work flow through bounding box technique. It looks for the feature that matched the letter given by remembering the concept of bounding box Yolo performs well with less time consumption.

\section{C.ResNet}

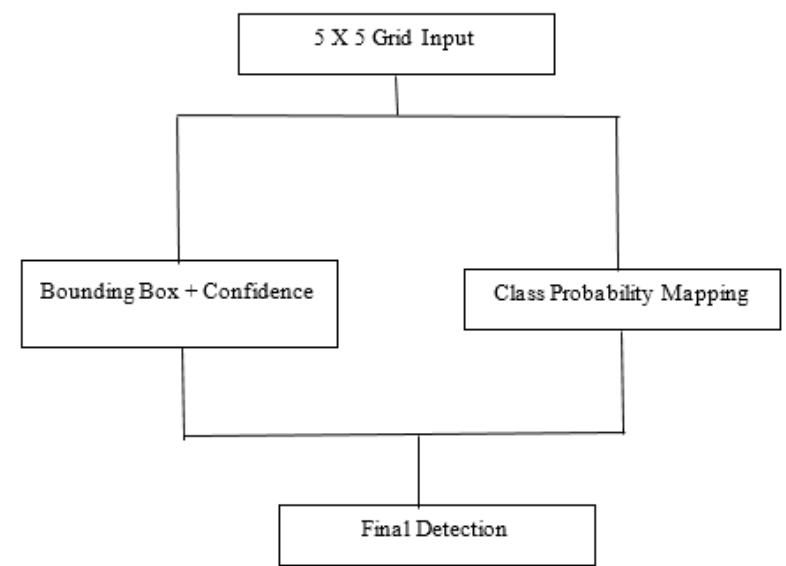

Fig.2 Yolo for character detection

ResNet has two processes mainly that used to compute the layer in parallel to recognize the object or character that follows up two important rules in the Residual block which by adding a new layer if any layer that would not hurt the model performance as the regularization that can be skipped over it cannot cause any changes in the model on the anther side by adding a new layer actually a useful presentation of the regularization the kernel will be non-zero to a load of the model that will increase the model efficiency inaccuracy. Fig. 3 explains the full stages of ResNet includes convolutional blocks and identify blocks. Identify block covers the standard block that is used in Resnet to correspond the input activation to have the same dimension to the output activation dimension for connecting that can be skipped all over the connection straight that goes to the last stage in the model. Getting Tamil character recognition in ResNet replaces the VGG16 by skipping more time with the same level of computation. It recognizes the letters by increasing the layers in the model by building them according to 5 stages deployed in ResNet fixed architecture. Convolution block is used where the input and the output dimension are not in the match; it introduces the Conv2D layer in the shortcut path.

\section{Exprimental Analysis}

\section{A. Dataset Description}


HP Labs India is providing a wide range of datasets that contains 60,000 images of isolated Tamil letters which were written by college students, adults, and school students with all kinds of letters in the Tamil language. The CNN architecture which needs the size of an image as same so here we are changing the input size as $256 * 256$ for all images to preprocessing purposes. In pre-processing, the images are color images (RGB); it also contains a separate channel value as 3 . As it was a grayscale image channel size will be in 1 .

The process that includes pre-processing that includes, set the path for the input images, to resize it into $256^{*} 256$ for use in CNN Fig.4 shows the dataset images to a particular Tamil vowel letter. Class labeling the data depending upon the work that has to be done for evaluation, the proposed system used Tamil vowels labeling with 13 classes i.e. 13 letters present in the Tamil vowels Table. 1 shows the class number for all the letters and Table. 2 shows the total number of images that are used for all the particular letters for training and testing the number count described the test count, train count, and random images count used for validating the dataset.

Table 1. Class Table

\begin{tabular}{|l|l|}
\hline Tamil Vowels & Class Labels \\
\hline அ & 0 \\
\hline ஆ & 1 \\
\hline ॠ & 2 \\
\hline ஈ & 3 \\
\hline ஊ & 4 \\
\hline எ & 5 \\
\hline ஏ & 6 \\
\hline ஐ & 7 \\
\hline ஓ & 8 \\
\hline ஓ & 9 \\
\hline ஓ & 10 \\
\hline$\square$ & 11 \\
\hline
\end{tabular}

Table 2.Image count

\begin{tabular}{|c|c|c|l|}
\hline Tamil vowel Letters & Test Count & Train Count & $\begin{array}{l}\text { Random } \\
\text { Image Count }\end{array}$ \\
\hline அ & 301 & 164 & 50 \\
\hline ஆ & 220 & 158 & 20 \\
\hline இ & 387 & 134 & 12 \\
\hline ஈ & 325 & 191 & 30 \\
\hline
\end{tabular}




\begin{tabular}{|l|l|l|l|}
\hline உ & 312 & 140 & 25 \\
\hline ஊ & 114 & 139 & 10 \\
\hline எ & 205 & 110 & 46 \\
\hline ஏ & 189 & 96 & 31 \\
\hline ஐ & 126 & 80 & 17 \\
\hline ஓ & 148 & 92 & 20 \\
\hline ஓௗ & 329 & 116 & 15 \\
\hline$\square$ & 329 & 64 & 15 \\
\hline TOTAL & 124 & 91 & 30 \\
\hline
\end{tabular}

\section{B. Experimental Setup}

The suggested techniques were thoroughly carried out on the Anaconda Navigator in order to process the implementation. Many packages that are used for character recognition in different environments are built-in with a separate environment that has been developed to perform the operation. The code is designed using the Python programming language. Jupyter notebook is used to run the kernel code. The proposed system consists of implementing CNN, OCR, Yolo, and Resnet with the same dataset of pre-processed results. 


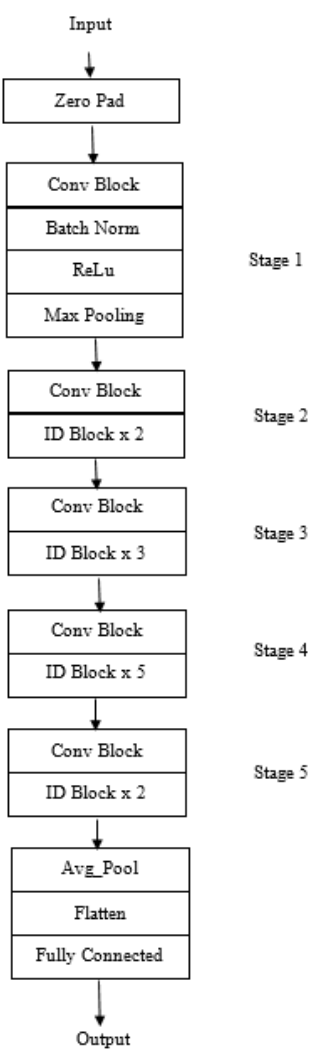

Fig.3 ResNet with Convolutional Block

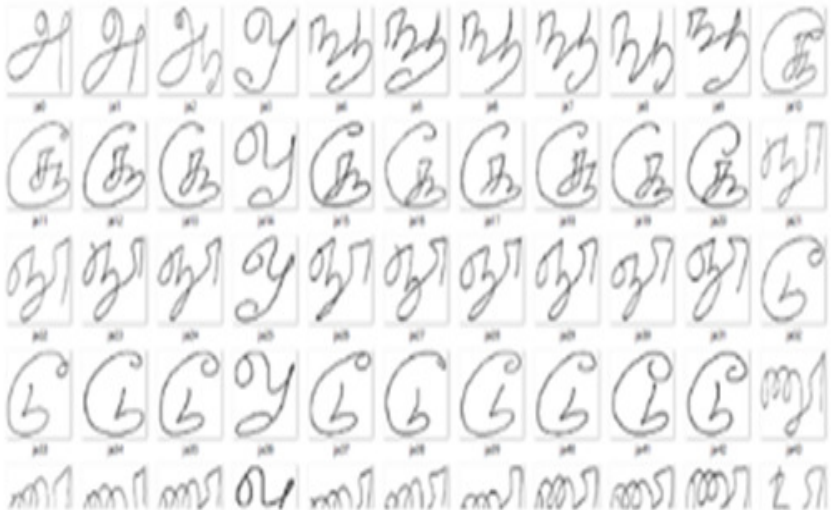

Fig 4.HP Labs India Dataset

CNN with Imagedatagenerator predicts the output with class labels where the input images of a raw is given into the model and it throws the output as the predicted value. The 
output layer with ReLu activation function calculates the matrix and gives the train and test accuracy.CNN works with high efficiency by consuming less time, Predicts well even if the images were given random, noise-containing images. OCR implants through pytesseract it fails to detect the Tamil characters present in the image and misses some letters while converting them into editable form. Yolo world is similar to CNN with time efficiency, as it is a pre-trained model it needs more data to train and test for a wide amount of data in the Tamil language. Feeding more data into the proposed Yolo system it takes a while to pre-train the model again.ResNet is a replacement for VGG16 it also holds the same complex problem as VGG16 by taking more time to compute and resulting in poor accuracy.

\section{Result Analysis}

Classifying the model efficient Confusion matrix is used to calculate the model performance. It works on the concept of comparing both the values of the actual value of a target with the predicted value of the equation. Equ. 1 states the Confusion matrix based on the target value in addition to the predicted value.

$$
\text { Accuracy }=\frac{T P+T N}{T P+F P+T N+F N}
$$

The above equation states the calculation for accuracy the terms indicated are TP -True positive, TN- True negative, FP-False positive, and FN- False-negative. The confusion matrix for Tamil character recognition predicts the accuracy rate on two errors, type 1 and type 2 . Type 1 is a false valued error where the value is negative but the model predicts positive. Opposite in Type 2, error where model predicted false and also the value is positive with negative prediction rate.Table 2 listed below illustrates the comparison of models performed for Tamil character recognition in deep learning models with their train and test accuracy.Fig.2 illustrates the model that performed the recognition and their respective accuracy obtained during the observation. $\mathrm{CNN}$ outperforms all the models performed with a high accuracy rate with good efficiency by predicting the result accurately than other models.

Table 3.Character Recognition Models with Accuracy

\begin{tabular}{|l|c|c|}
\hline Character Recognition Models & $\begin{array}{l}\text { Train } \\
\text { Accuracy }\end{array}$ & Test Accuracy \\
\hline CNN & $98 \%$ & $92 \%$ \\
\hline Yolo & $89 \%$ & $82 \%$ \\
\hline ResNet & $81 \%$ & $79 \%$ \\
\hline
\end{tabular}

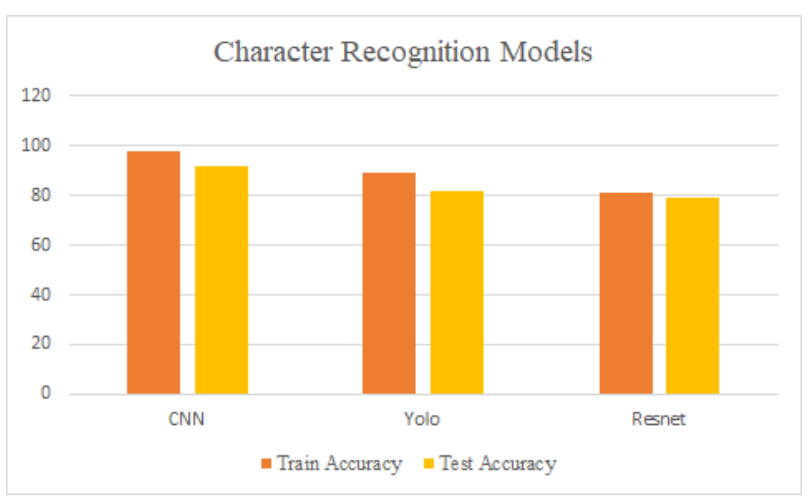




\section{Conclusion}

\section{Fig. 5. Model Comparison Graph}

The proposed work for Tamil character recognition demonstrates that $\mathrm{CNN}$ is the best fit model for continuing the work where input data was provided in image form to extract the letters and convert them into editable form. Yolo and ResNet are functional, but when compared to $\mathrm{CNN}$, it outperforms by being more efficient at adding data, predicting letters with noise, blurred images, and titled images The proposed system covers alphabets of Tamil vowels in isolated form of separate letters not in words; future work will focus on increasing the dataset for extension of all Tamil dictionary words and Corpus. To train the model for recognition of Tamil letters and to convert them into editable form, the dataset will extend to include Tamil words and remaining letters in the Tamil language.

\section{References}

[1] Ramanan, M., Ramanan, A. and Charles, E.Y.A., , August. A hybrid decision tree for printed Tamil character recognition using SVMs. In 2015 Fifteenth International Conference on Advances in ICT for Emerging Regions (ICTer) (pp. 176-181). IEEE.

[2] Ishwarya, M.V. An Improved Online Tamil Character Recognition Using Neural Networks. International Journal of Advanced Science and Technology, 42, pp.1-10

[3] Balci, B., Saadati, D. and Shiferaw, D., Handwritten text recognition using deep learning. CS231n: Convolutional Neural Networks for Visual Recognition, Stanford University, Course Project Report, Springer, pp.752-759.

[4] N.Sasipriya, K.Abirami, G.Banupriya, S.Dhivya.International Journal of Advanced Science and Technology Vol. 29, No. 3s, (2020), pp. 981-987.

[5] Ishwarya, M.V.An Improved Online Tamil Character Recognition Using Neural Networks. International Journal of Advanced Science and Technology, 42, pp.1-10.

[6] Gautam, N., Chai, S.S. and Jose, J., Recognition of Brahmi Words by Using Deep Convolutional Neural Networks

[7] Ramanan, M., Ramanan, A. and Charles, E.Y.A.,, August. A hybrid decision tree for printed Tamil character recognition using SVMs. In 2015 Fifteenth International Conference on Advances in ICT for Emerging Regions (ICTer) (pp. 176-181). IEEE

[8] Ramanan, M., Ramanan, A. and Charles, E.Y.A., A hybrid decision tree for printed Tamil character recognition using SVMs. In 2015 Fifteenth International Conference on Advances

[9] Giridhar, L., Dharani, A. and Guruviah, V., A Novel Approach to OCR using Image Recognition based Classification for Ancient Tamil Inscriptions in Temples. arXiv preprint arXiv:1907.04917.

[10] Wahi, A.D.A., Sundaramurthy, B.M.S. and Poovizhi, C.P., Handwritten Tamil Character Recognition using Moments.

[11] Pragathi, M.A., Priyadarshini, K., Saveetha, S., Banu, A.S. and Aarif, K.M., 2019, March. Handwritten Tamil Character Recognition UsingDeep Learning. In 2019 International Conference on Vision Towards Emerging Trends in Communication and Networking (ViTECoN) (pp. 1-5). IEEE. 
[12] AnupriyaGogna and Angshul Majumdar. . "Hyperspectral image classification based on structured sparse logistic regression and three dimensional wavelet texture features." IEEE Transactions on Geoscience and Remote Sensing 51, no. 4 (2013): 2276-2291

[13] Lei Chea,b , Xiaoping Yanga , Liang Wanga. 0141-9331/@ 2020 Elsevie

[14] Laroca, R., Severo, E., Zanlorenzi, L.A., Oliveira, L.S., Gonçalves, G.R., Schwartz, W.R. and Menotti, D.,2018, July. A robust real-time automatic license plate recognition based on the YOLO detector. In 2018International Joint Conference on Neural Networks (IJCNN) (pp. 1-10). IEEE.

[15] Mhaskar, P., Trivedi, P., Mandal, S. and Mitra, S.K., 2021. Handwritten Digit Recognition Using Bayesian ResNet. SN Computer Science, 2(5), pp.1-10.

[16] Jijina, G.O., Mohana Priya, R., Solainayagi, P." FPGA Realization of Reconfigurable DA-Based Digital FIR Filter Using DRPPG and MCSA Techniques", Lecture Notes in Networks and Systems, 2021, 179 LNNS, pp. 527-544

[17] SheebaPercis, E., Nalini, A., Jijina, G.O.,Selvarani, N., Sendilvelan, S."Stability analysis of dedicated green energy corridors and enhancement of renewable energy evacuation" IOP Conference Series: Materials Science and Engineering, 2020, 993(1), 012071.

[18] Nalini, A., SheebaPercis, E., Jijina, G.O., Raja, M., Sendilvelan, S."Supervisory protection scheme for a wide area $400 \mathrm{KV}$ power network", IOP Conference Series: Materials Science and Engineering, 2020, 993(1), 012072 